\title{
Effect of Box Microspring Sizes on Nonlinear Deformation
}

\author{
Cho-Chun Wu, Meng-Ju Lin ${ }^{1}$ and Rongshun Chen* \\ Department of Power Mechanical Engineering, National Tsing Hua University, \\ Hsinchu City 30013, Taiwan \\ ${ }^{1}$ Department of Mechanical \& Computer-Aided Engineering, \\ Feng Chia University, Taichung, Taiwan
}

(Received August 15, 2008; accepted December 15, 2008)

Key words: $\quad$ box microspring, nonlinear deformation, spring constant, size effect

Microsprings are often used in micro-electro-mechanical system (MEMS) actuators to transmit force and to restore its original position by its spring force after a movement. Owing to its high stiffness and good capability of resisting lateral forces, the box microspring has the advantages of resisting induced transverse forces and preventing lateral deformation over other microsprings. For better operation, the nonlinear behavior of the microspring should be avoided when the spring is used in MEMS devices. Microspring size can significantly affect microspring performance. In this paper, we report on the effect of box microspring size on the nonlinear deformation of the microspring. The width $(W)$ of the vertical beam of rectangular frames, microspring thickness $(T)$, the width $(B)$ of the horizontal beam of rectangular frames, and the spring number $(N)$ of the box microspring are used as parameters to investigate the effect of box microspring size on nonlinear force. The finite element software COMSOL Multiphysics is used as the simulation tool. From the simulation results, the linear spring constant $k$ and cubic spring constant $k_{3}$ are determined and expressed in terms of $T, B, W$, and $N$ by the regression analytical method. The simulation results of this work can be used to design a microspring in an actuator such that nonlinear deformation is avoided.

\section{Introduction}

Microsprings are often used in micro-electro-mechanical system (MEMS) actuators to transmit force and recover its original position by spring force after a movement. They can also be used to measure forces of microactuators ${ }^{(1)}$ and become the pivot of MEMS to connect to other components. ${ }^{(2-4)}$ There are two types of microspring often used:(5) one is called the box microspring and the other is called the zigzag (serpentine) microspring. ${ }^{(6)}$ Under the same conditions, the former has a larger spring constant $k$ than the latter. Owing to its high stiffness and good capability of resisting lateral forces,

${ }^{*}$ Corresponding author: e-mail: rchen@pme.nthu.edu.tw 
the box microspring has the advantages of resisting induced transverse forces and preventing lateral deformation over the zigzag microspring. In other words, to maintain balance in the lateral direction, the box microspring is more effective than other types of microspring. Moreover, the box microspring has better controllability under applied force. ${ }^{(7)}$

For better operation, the nonlinear behavior of the microspring should be avoided when the spring is utilized in MEMS devices. Microspring size can significantly affect microspring performance, such as displacement, deformation, and equivalent spring constant. In this paper, we report on the effect of box microspring size on microspring deformation, particularly for nonlinear behavior. The width $(W)$ of the vertical beam of rectangular frames, microspring thickness $(T)$, the width $(B)$ of the horizontal beam of rectangular frames, and the spring number $(N)$ of the box microspring are used as the parameters for determining the relationship between box microspring size and nonlinear force. On the basis of variations in box microspring size, nonlinear deformations are investigated using the finite element software COMSOL Multiphysics as the simulation tool. Moreover, from the simulation results, the linear spring constant $k$ and cubic spring constant $k_{3}$ are determined and expressed in terms of $T, B, W$, and $N$ by the regression analytical method. The results of this work can be used to design a microspring in an actuator such that nonlinear deformation is avoided.

\section{Numerical Analysis on Box Microspring}

The spring constant of a linear spring can be determined from Hook's law. However, if the applied force is large, the spring may result in nonlinear deformation and can be expressed as the Duffing equation, ${ }^{(8)}$ in which the elastic restoring force $F$ can be simplified as

$$
F=k x+k_{3} x^{3}
$$

where $x$ is the displacement of the spring, and $k$ and $k_{3}$ are the linear and cubic elastic constants of the spring, respectively.

Owing to the complex coupling of mechanics behavior, it is very difficult to obtain the analytical solution of nonlinear deformation for a box microspring. Therefore, a numerical method is used to investigate the displacements of the box microspring under various applied forces. The finite element software COMSOL Multiphysics is used as the analysis tool. To compare deformations, linear and nonlinear modes are introduced in the numerical simulations.

It is assumed that the microspring is made of polysilicon with Young's modulus of 169 Gpa. The schematic of the box microspring with four geometric sizes is shown in Fig. 1. Because the sizes of MEMS devices are often limited, the length of the box microspring will be constrained for practical use. In our design, the horizontal beam length $(L)$ and the total length of the box microspring are maintained at 200 and $670 \mu \mathrm{m}$, respectively, throughout the entire simulation. $B, T$, and $W$ are considered as the size parameters that affect the nonlinear displacement of the box microspring, as shown in Fig. 1. 

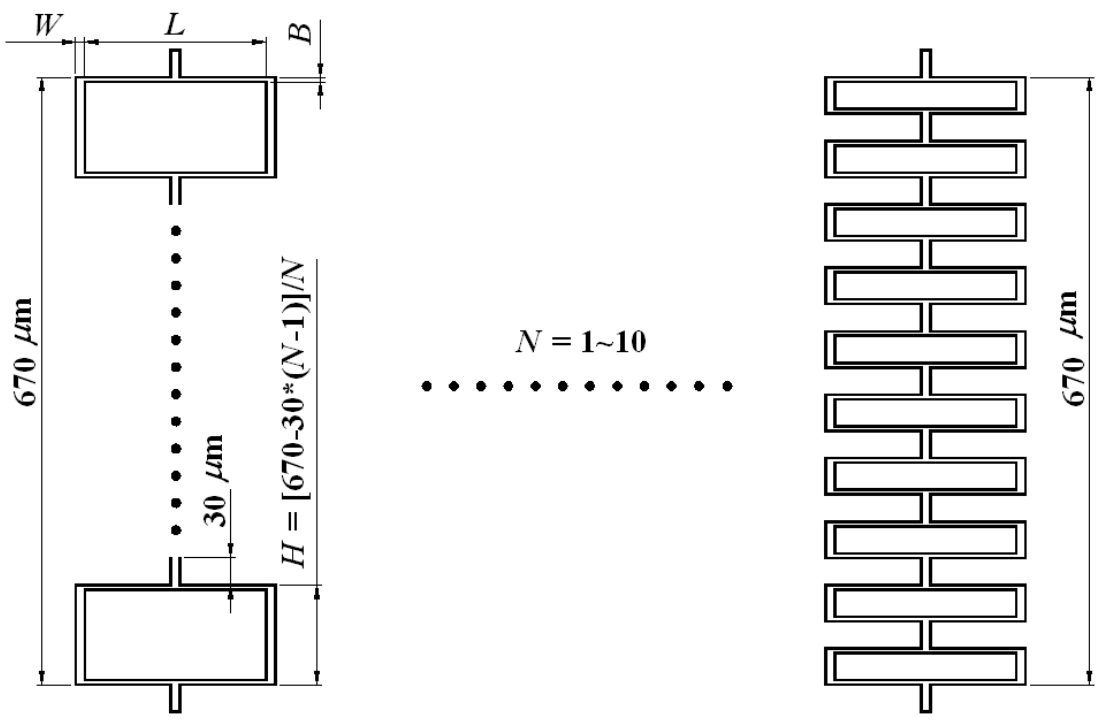

Fig. 1. Schematic of box microspring with the size parameters.

Another parameter called the density of spring number, $N$, defined as the number of turns within a constant total spring length, also affects the nonlinear displacement.

When the tensile and compressive forces are applied, the box microspring has a different deflection behavior, as shown in Figs. 2(a) and 2(b), respectively, where $W=$ $10 \mu \mathrm{m}, B=5 \mu \mathrm{m}, T=2 \mu \mathrm{m}, L=200 \mu \mathrm{m}$, the spring number of turns $N=1$, and the total length of the microspring is $670 \mu \mathrm{m}$. Because tensile force is used, the four beams of the box microspring are all elongated owing to the bending moment, as shown in Fig. 2(a). However, the directions of the displacements of the horizontal and vertical beams are different in the figure. On the other hand, when compressive force is applied to the box microspring, the lengths of the four beams are still elongated owing to the bending moment. However, the horizontal and vertical deformations are in the same direction, as shown in Fig. 2(b). By using the same size and $N$ as those in Fig. 2, Fig. 3 shows the displacements of the box microspring under compression and tension, in which the compressive box microspring has a larger displacement than the tensile one. Particularly, when the applied force is large, the difference in displacement can be up to six times. Consequently, buckling may occur easily in the microspring under compression because of its large displacement. Because the microsping under compression will result in a large displacement and may not be well controlled in practice, in this work, we only consider the deformation of the box microspring under tension. The effect of the beam size of the box microspring on nonlinear deformation is investigated.

The static analysis of plane strain of structural mechanics module for nonlinear and large deformations is employed. Under different forces, displacement can be obtained using COMSOL Multiphysics. To understand the nonlinear behavior of a box microspring, it is necessary to find the starting point of nonlinear deformation; 


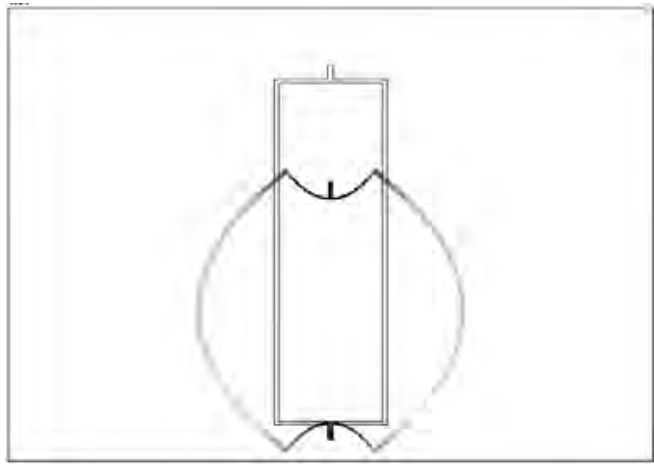

(a)

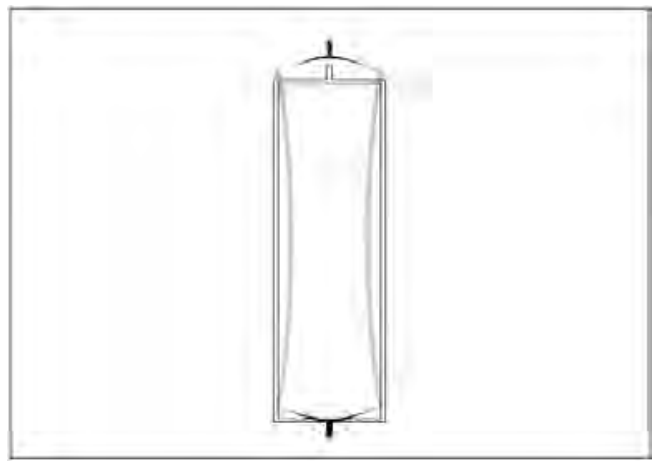

(b)

Fig. 2. Deformations of box microspring under (a) compressive force and (b) tensile force.

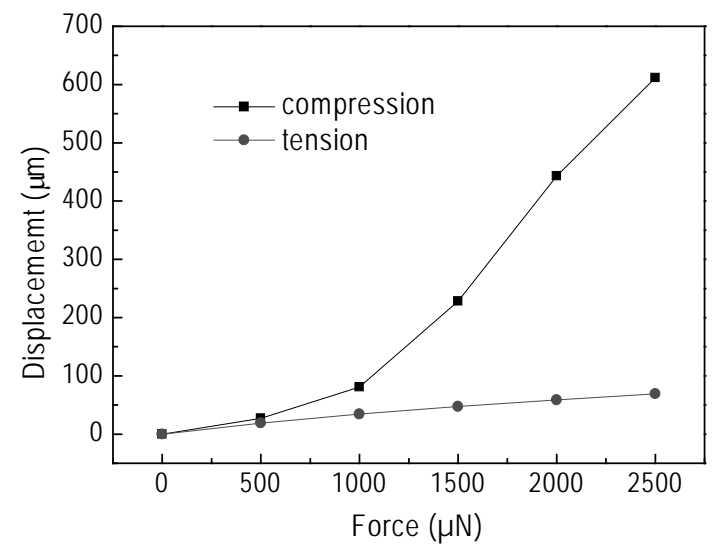

Fig. 3. Displacements of box microspring under compression and tension.

unfortunately, it is very difficult to find. We, therefore, define a "critical" point of nonlinear deformation as follows. If the linear deformation has a magnitude of $90 \%$, it is defined as the "critical" point of nonlinear deformation behavior and is regarded as the beginning of the nonlinear behavior of the box microspring. In this work, the force when the critical point occurred is called the critical nonlinear force for convenience.

Figure 4 shows the simulated results of linear and nonlinear deformations for different applied forces, where the microspring is pulled under tensile force. The sizes used in the simulations in Fig. 4 are the same as those in Fig. 3, but $N=10$ in Fig. 4. Three curves (lines), namely, linear, nonlinear, and 0.9*linear, are presented in Fig. 4, where 0.9*linear denotes that the magnitude of this line reaches $90 \%$ of its original linear displacement. 


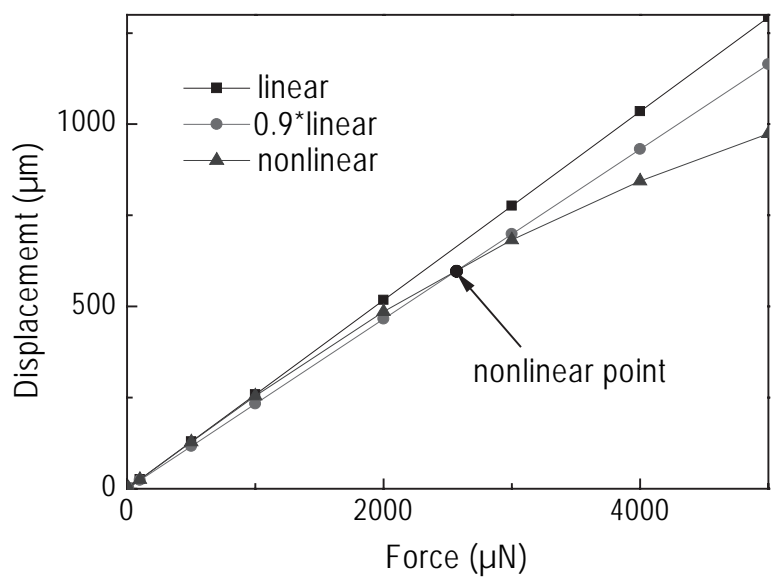

Fig. 4. Simulated results of linear, 0.9*linear, and nonlinear deformations for different applied forces, where $W=10 \mu \mathrm{m}, B=5 \mu \mathrm{m}, T=2 \mu \mathrm{m}, L=200 \mu \mathrm{m}, N=10$, and the total length the microspring is $670 \mu \mathrm{m}$.

Because the applied force is small, the linear and nonlinear deformations only show a small difference. However, as applied force increases, the difference between the linear and nonlinear deformations becomes significant, in which the nonlinear deformation is a curve, bending with decreasing slope and having a magnitude less than that of the linear deformation. The intersection point of the $90 \%$ linear deformation line and nonlinear deformation curve, denoted as the nonlinear point in Fig. 4, is regarded as the beginning of the nonlinear deformation behavior in this study.

In the first group of simulations, shown from Figs. 5 to 8 , the sizes of $B$ and $T$ can be varied from 1 to $5 \mu \mathrm{m}$, and $N$ from 1 to 10 , while $W$ is maintained at $10 \mu \mathrm{m}$. In the simulations for determining the critical nonlinear force, the size parameters $B, T$, and $W$, the number of turns $N$, and trial force were given first. By carrying out linear and nonlinear analyses using COMSOL Multiphysics, linear and nonlinear displacements are obtained. The critical nonlinear force will be found if the nonlinear displacement is about $90 \%$ of the linear displacement. By obtaining the critical nonlinear forces under different size parameters $B, T$, and $W$, and number of turns $N$, the relationship between nonlinearity and the parameters can be found. In the second group of simulations, shown from Figs. 9 to $11, B, T$, and $W$ can be varied from 1 to $5 \mu \mathrm{m}$, and $N$ from 1 to 10 . Note that the total microspring length is $670 \mu \mathrm{m}$; hence, the heights of the box microspring, $H$, are different for different numbers of turns. If the length connecting two adjacent rectangular boxes is $30 \mu \mathrm{m}, H$ can be calculated as $H=[670-30 \times(N-1)] / N$. For example, if $N=5, H=110 \mu \mathrm{m}$. The larger the $N$, the smaller the $H$. 


\section{Results and Discussion}

Figure 5 shows the relationship between critical nonlinear force and the width of the horizontal beam of rectangular frames $B$ for different $N$ s. For a fixed $N$ (except for $N=1$ ), critical nonlinear force increases rapidly if $B$ is large, while the increase is slow if $B$ is small, as shown in Fig. 5 . The simulated results also indicated that critical nonlinear force remains almost the same when $N \geq 4$ and $B \leq 3 \mu \mathrm{m}$. Furthermore, critical nonlinear forces significantly differ for $1<N \leq 4$ with $B=4-5 \mu \mathrm{m}$. Figure 6 shows the relationship between critical nonlinear force and the number of turns $N$ under different Bs. From Fig. 6, when $B \geq 4 \mu \mathrm{m}$, critical nonlinear force increases rapidly as $N$ increases. At $B=3 \mu \mathrm{m}$, critical nonlinear force shows no increase at $N \geq 2$; it eventually approaches a limit of about $1500 \mu \mathrm{N}$. For $B=1$ and $2 \mu \mathrm{m}$, there is no gain of the critical nonlinear force from $N=1-10$. It is thus concluded that if a box microspring is used to

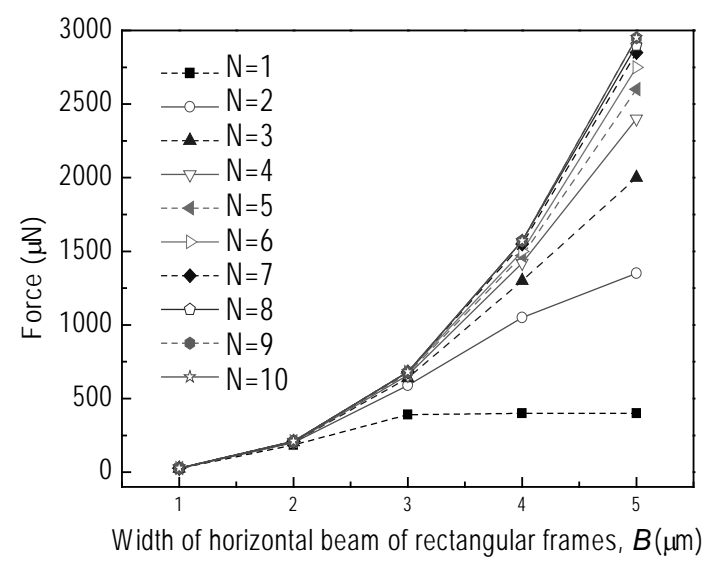

Fig. 5. Relationship between critical nonlinear force and $B$, under different numbers of turns $N$ with $W=10 \mu \mathrm{m}, T=2 \mu \mathrm{m}$, and $L=200 \mu \mathrm{m}$.

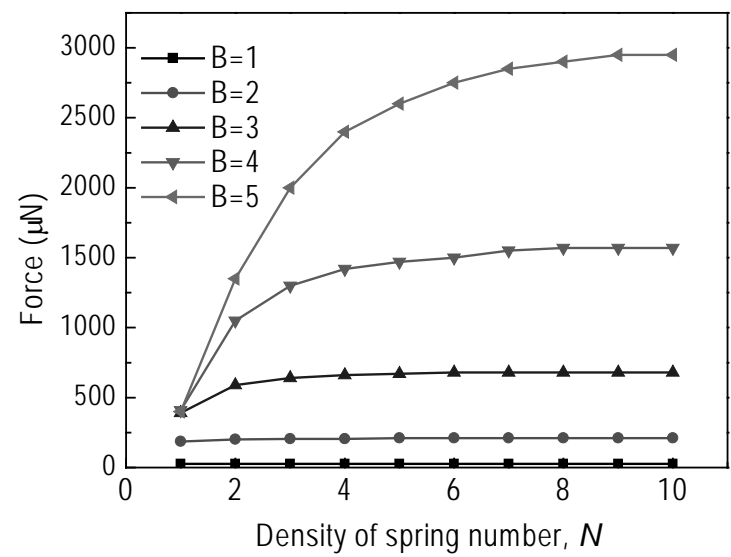

Fig. 6. Relationship between critical nonlinear force and number of turns $N$ for different Bs with $W=10 \mu \mathrm{m}, T=2 \mu \mathrm{m}$, and $L=200 \mu \mathrm{m}$. 
resist the nonlinear behavior, a larger $B$ can extend the point of the nonlinear force and its nonlinear behavior can be avoided.

As seen in Figs. 5 and 6, it is very clear that the increase in critical nonlinear force is quite different from the effects of $B$ and $N$. If $N$ is fixed, the increasing $B$ makes the critical nonlinear force an exponential curve (Fig. 5); if $B$ is fixed, the critical nonlinear force increases following a logarithm-like curve when $N$ is increasing (Fig. 6).

The effect of $T$ on critical nonlinear force is shown in Fig. 7 for different $N$ s, where the critical nonlinear force increases linearly as $T$ increases at the same $N$. Notice that the slope ratio is larger when $N$ is larger, and the critical nonlinear force has a smaller difference, in comparison with the smaller $N$, from $N=5$ to 10 for the same $T$. Figure 8 shows the relationship between critical nonlinear force and $N$ under different Ts. The curve of critical nonlinear force looks like a logarithmically growing curve as $N$

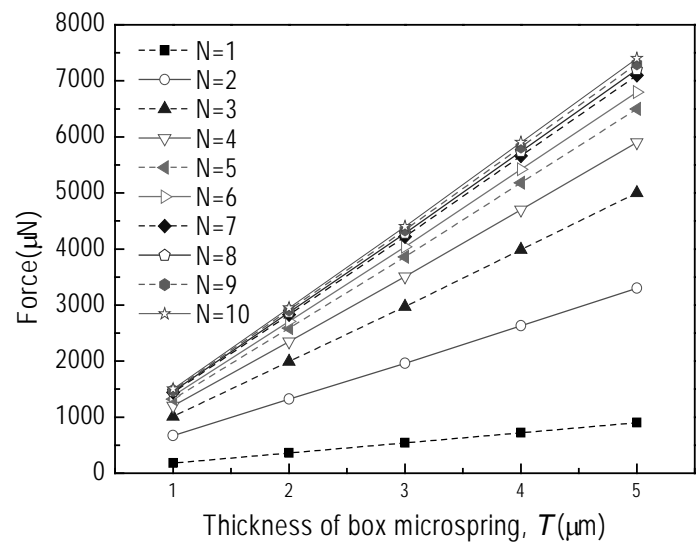

Fig. 7. Relationship between critical nonlinear force and $T$, under different numbers of turns $N$ with $W=10 \mu \mathrm{m}, T=5 \mu \mathrm{m}$, and $L=200 \mu \mathrm{m}$.

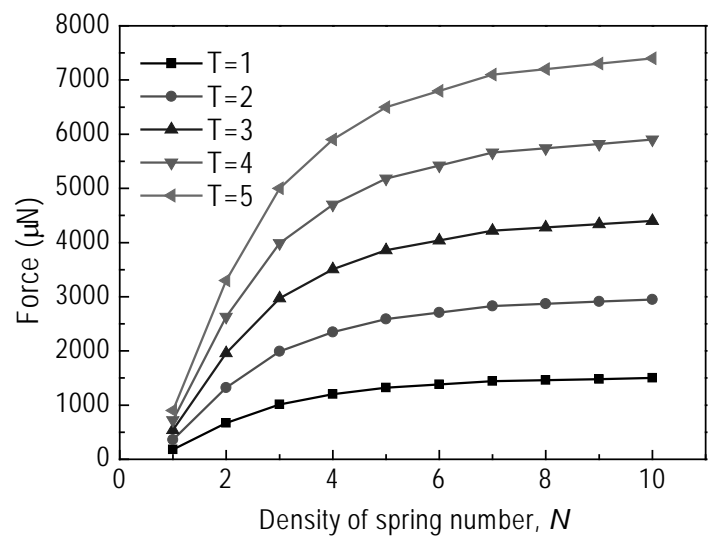

Fig. 8. Relationship between critical nonlinear force and number of turns $N$, under different $T \mathrm{~s}$ with $W=10 \mu \mathrm{m}, T=5 \mu \mathrm{m}$, and $L=200 \mu \mathrm{m}$. 
increases for a specific $T$. However, for higher $T$, the increase rate of critical nonlinear force is larger than that for smaller T. From the results in Figs. 6 and 8, it is found that the relationship between critical nonlinear force and the number of turns $N$ is very similar for various Bs and Ts. Nevertheless, the critical nonlinear force in Fig. 8 is much larger than that in Fig. 6 since $T$ is maintained at $2 \mu \mathrm{m}$ in Fig. 6. Consequently, the nonlinear behavior hardly happens for a thicker microspring and a larger number of turns, as well as for a larger width of the box microspring.

For the analysis of the linear spring constant $k$ and the cubic spring constant $k_{3}$, FEM simulation was performed to determine the relationship between critical nonlinear force and displacement, under different spring numbers of turns $N$. Regressive analysis is used to obtain the linear spring constant $k$ and the cubic spring constant $k_{3}$ using eq. (1). These simulations are performed using the large deformation option module of COMSOL Multiphysics.

The thickness of the box microspring, $T$, versus $k$ and $k_{3}$ for different $N$ s is shown in Figs. 9(a) and 9(b), respectively, where $B=5 \mu \mathrm{m}$ and $W$ and $L$ are the same as the previous values. It indicates that for the same $N, k$ decreases with increasing $T$. Also, the larger the $N$, the larger the $k$ for the same $T$, whereas $k$ decays quicker if $N$ is larger, as shown in Fig. 9(a). Similarly to Fig. 9(a), Fig. 9(b) shows that the larger $N$ results in a larger magnitude of $k_{3}$, which decays quicker if $N$ is larger. However, the magnitude of $k_{3}$ is smaller than that of $k$, and has a negative sign. At $T=2 \mu \mathrm{m}$, the width $B$ versus $k$ and $k_{3}$ for different $N$ s is shown in Figs. 10(a) and 10(b), respectively. The nonlinear behaviors shown in Figs. 10(a) and 10(b) are very similar to those shown in Figs. 9(a) and 9(b), respectively, where the role of $T$ in Fig. 9 is replaced by $B$ in Fig. 10, and will not be described again for simplicity. For $B=5 \mu \mathrm{m}, T=2 \mu \mathrm{m}$, the values of $k$ and $k_{3}$ versus $W$ for different $N$ s are shown in Figs. 11(a) and 11(b), respectively, which have almost the same nonlinear behaviors as those shown in Figs. 9 and 10.

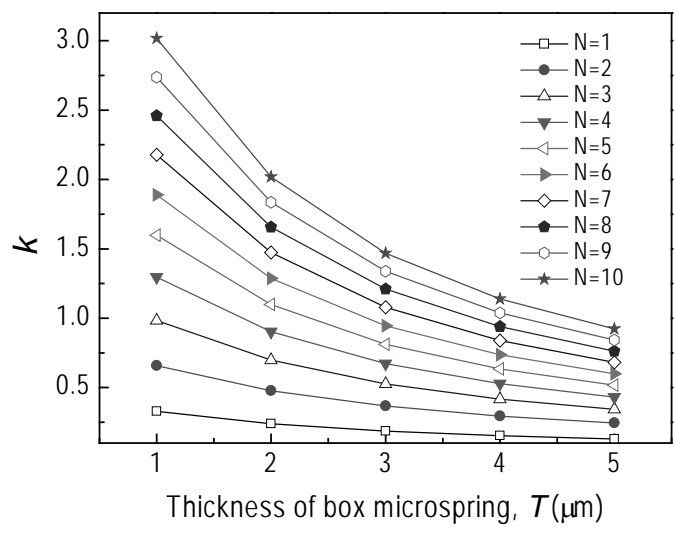

(a)

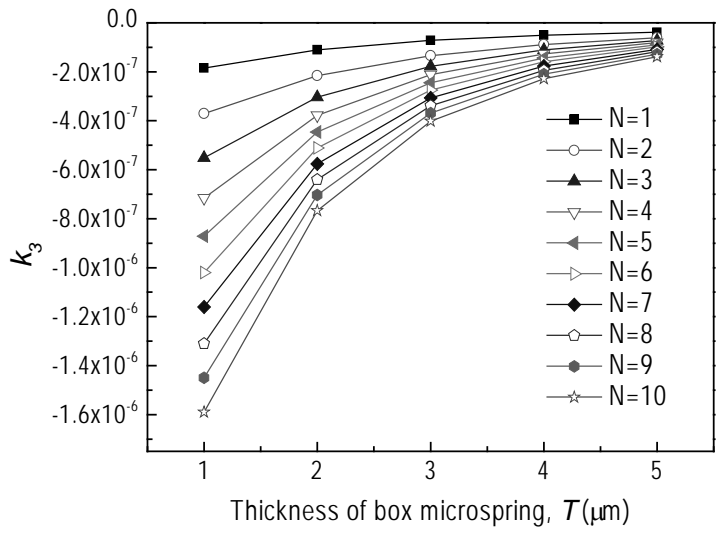

(b)

Fig. 9. Relationship between $T$ and spring constants (a) $k$ and (b) $k_{3}$, under different numbers of turns $N$ with $W=10 \mu \mathrm{m}, B=5 \mu \mathrm{m}$, and $L=200 \mu \mathrm{m}$. 


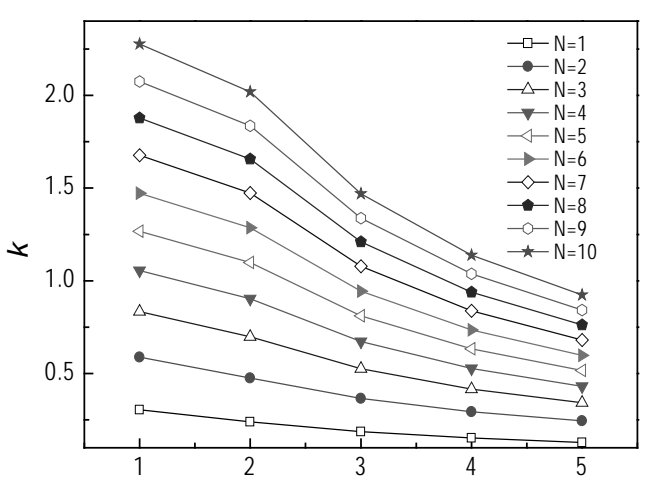

Width of horizontal beam of rectangular frames, $B(\mu \mathrm{m})$

(a)

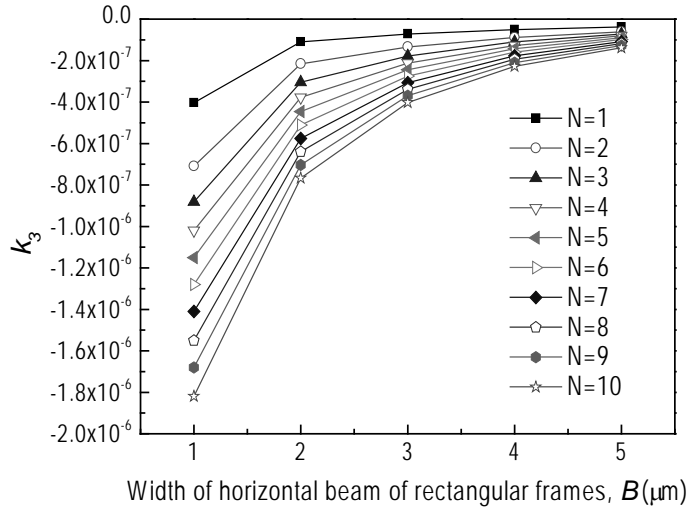

(b)

Fig. 10. Relationship between $B$ and spring constants (a) $k$ and (b) $k_{3}$, under different numbers of turns $N$ with $W=10 \mu \mathrm{m}, T=2 \mu \mathrm{m}$, and $L=200 \mu \mathrm{m}$.

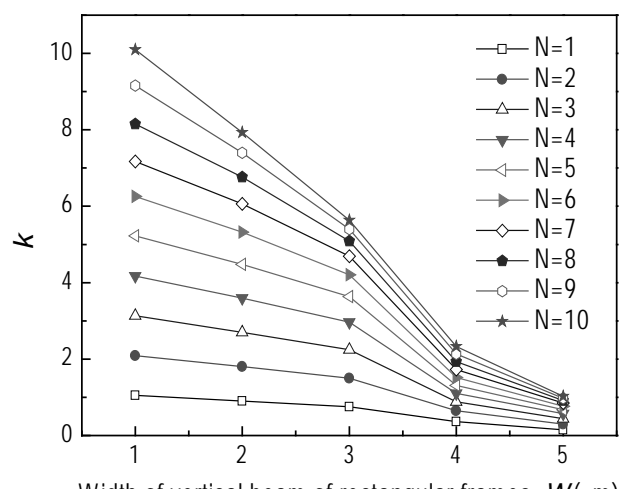

Width of vertical beam of rectangular frames, $W(\mu \mathrm{m})$

(a)

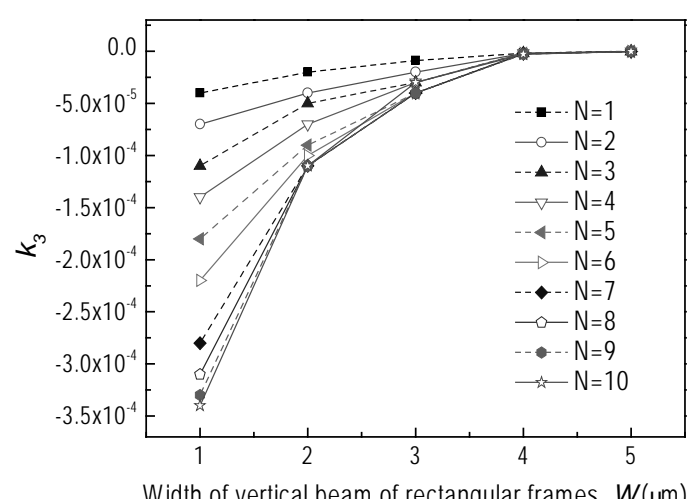

(b)

Fig. 11. Relationship between $W$ and spring constants (a) $k$ and (b) $k_{3}$, under different numbers of turns $N$ with $B=5 \mu \mathrm{m}, T=2 \mu \mathrm{m}$, and $L=200 \mu \mathrm{m}$.

By using the simulation results in Figs. 9, 10, and 11, the spring constants $k$ and $k_{3}$ in terms of $T, B, W$, and $N$ can be determined by employing the regressive analytical method. The derived equations of $k$ and $k_{3}$ can be expressed as

$$
\begin{gathered}
k=21.86 T^{-0.57} B^{-0.21} W^{-1.7} N^{0.87}, \\
k_{3}=8.183 \times 10^{-4} T^{-0.93} B^{-0.95} W^{-3.13} N^{0.66} .
\end{gathered}
$$




\section{Conclusion}

In this paper, we report the effect of box microspring size on the nonlinear deformation of a microspring. The width $(W)$ of the vertical beam of rectangular frames, microspring thickness $(T)$, the width $(B)$ of the horizontal beam of rectangular frames, and the spring number $(N)$ of the box microspring are used as parameters to investigate the effect of box microspring size on nonlinear force. The finite element software COMSOL Multiphysics is used as the simulation tool. From the simulation results, the linear spring constant $k$ and cubic spring constant $k_{3}$ are determined and expressed in terms of $T, B, W$, and $N$ by the regression analytical method. It is concluded that a larger $B$ of a box microspring can extend the point of nonlinear force and its nonlinear behavior can be avoided. Furthermore, the nonlinear behavior may hardly happen in the case of a thick microspring, a large number of turns, and a large box microspring width.

The simulated results of this work can be used to design a microspring in an actuator such that the nonlinear deformation is avoided.

\section{References}

1 L. Li and D. G. Uttamchandani: Design, Test, Integration, and Packaging of MEMS/MOEMS 4755 (2002) 394.

2 C. Huang, C. Christophorou, K. Najafi, A. Naguib and H. M. Nagib: Journal of Microelectromechanical Systems 11 (2002) 222.

3 H. Xie and G. K. Fedder: IEEE Sensors Journal 3 (2003) 622.

4 D. Peroulis, S. P. Pacheco, K. Sarabandi and L. P. B. Katehi: IEEE Transactions on Microwave Theory and Techniques 51 (2003) 59.

5 V. Gupta and T. Mukherjee: International Conference on Modeling and Simulation of Microsystems Semiconductors (2000) p. 150.

6 Z. Ningning, A. Agogino and K. Pister: ASME DETC 2002 Design Automation (ASME, Montreal, Canada, 2002).

7 C. C. Wu, R. Chen and M. J. Lin: Advanced Materials Research 33 (2008) 975.

8 J. J. Stoker: Nonlinear Vibrations (New York University Press, New York, 1941). 